\title{
Medication adherence interventions and outcomes: an overview of systematic reviews
}

\author{
Nina C Wilhelmsen, ${ }^{1}$ Tommy Eriksson ${ }^{1,2}$
}

\begin{abstract}
- Additional material is published online only. To view please visit the journal online (http://dx.doi.org/ 10.1136/ejhpharm-2018001725).
\end{abstract} Molecular Medicine, Faculty of Medicine and Health Sciences, Norwegian University of Science and Technology (NTNU), Trondheim, Norway ${ }^{2}$ Department of Biomedical Sciences, Faculty of Health and Society, Malmö University,

\section{Correspondence to} Pharmacology, Laboratory Medicine, Lund University, Lund 22100 , Sweden; tommy. eriksson@mah.se

Received 17 August 2018 Revised 1 October 2018

Accepted 4 October 2018 Published Online First 16 November 2018

EAHP Statement 6: Education and Research.
${ }^{1}$ Department of Clinical and Malmö, Sweden Dr Tommy Eriksson, Clinical

\begin{abstract}
Objective To present evidence for healthcare-provided medication adherence interventions on clinical, economic and humanistic outcomes among patients.

Methods Literature search of systematic reviews in Medline, Embase and CINAHL (2007-2017), validation of quality using A Measurement Tool to Assess

Systematic Reviews (AMSTAR) 2 and Preferred Reporting Items for Systematic Reviews and Meta-Analyses questionnaires and, finally, extraction, combination and tabulation of results for included studies.

Results From eight systematic reviews with medium to high AMSTAR 2 score, 37 randomised controlled studies involving 28600 participants were extracted. Patient education and counselling showed some positive effects on medication adherence. Patient education also showed some positive effects on morbidity, healthcare utilities and patient satisfaction. Counselling had some benefit on mortality and healthcare utilisation. Simplifying doses was shown to have some benefit on morbidity and patient satisfaction. Interventions delivered by pharmacists and nurses showed a better result in improving adherence and outcomes than interventions delivered by general practitioners.

Conclusions Some interventions were found to have positive effect on adherence and outcomes, but no single strategy showed improvement in all settings. For future research patients should be screened for non-adherence to reveal both if they are non-adherent and type of nonadherence, as well as bigger sample sizes and longer duration of follow-up.
\end{abstract}

\section{INTRODUCTION}

Every new method of drug treatment being implemented in healthcare has to be proven effective, both when it comes to costs and clinical effects. It has preferred to use the most efficient intervention that provides a maximum of benefit for a minimum of costs. Providing good healthcare service is dependent on the utilisation of available knowledge and experience, and the use of new knowledge simultaneously as outdated practice ceases. In addition, there should be a local, regional or national agreement about what is considered good or preferred service performance.

Systematic reviews are one of the key tools for (C) European Association of Hospital Pharmacists 2019. No commercial re-use. See rights and permissions. Published by BMJ.

To cite: Wilhelmsen NC, Eriksson T. Eur J Hosp Pharm 2019;26:187-192. achieving evidence-based healthcare for providers and healthcare decision makers, so the systematic reviews should provide the accurate and the best available evidence on the topic of interest. ${ }^{1}$ Systematic reviews of several randomised trials are seen as the 'gold standard'2 and provide the highest level of evidence. ${ }^{3}$
Healthcare providers are responsible for choosing the right treatment for each patient, and that requires professional skill in searching for, assessing and using evidence-based information in clinical practice. ${ }^{4}$ This approach is called evidencebased medicine, meaning that provided healthcare is supported by high-quality evidence, does more good than harm and provides the greatest benefit for the patients. ${ }^{5}$

Drug therapy is important in protecting, maintaining and restoring people's health, ${ }^{6}$ and has therefore an important priority in the resources used in healthcare. Since medications are critical to prevent and treat disease, ${ }^{7}$ we must ask what happens when medications are not taken properly, that is, when adherence is low.

Adherence is defined as: 'The extent to which the patient's behaviour matches agreed recommendations from the prescriber. ${ }^{8}$ WHO stated in 2003 that adherence to long-term therapies was as low as $50 \%$ in the general population, and even much lower in low/middle-income countries. ${ }^{9}$ A Cochrane review stated that people who are prescribed medication for self-administration only take half of their prescribed dose, and many stop taking their medication entirely without informing their provider. ${ }^{10}$ Many patients who continue taking their medication do not do it consistently as prescribed. There is also an increasing number of self-administered treatments available, making improved adherence even more important.

Adherence to medication is an essential component of health outcome, ${ }^{9} 11$ so by increasing medication adherence we can also improve patient outcomes. ${ }^{10}$ Even the most carefully chosen and optimal medication can be rendered ineffective by insufficient adherence. ${ }^{9} 11$ Failure of medication adherence leads to substantial worsening of disease, death and increased healthcare costs. In other words, non-adherence affects both the individual patients and the healthcare system. ${ }^{9}{ }^{12}$ Studies have shown that patients with good adherence to treatment had both a lower rate of mortality ${ }^{13-15}$ and a lower rate of hospital admissions than patients with poor adherence to treatment. ${ }^{13} 1617$ WHO states, 'increasing the effectiveness of adherence interventions may have a far greater impact on the health of the population than any improvement in specific medical treatments." 9

Donabedian's theoretical framework for evaluating quality of care consists of three approaches: structure, process and outcome. The structure represents the conditions under which care is provided, the process represents the activities that constitute healthcare and the outcome represents the changes in the health status. ${ }^{18}$ It is suggested 


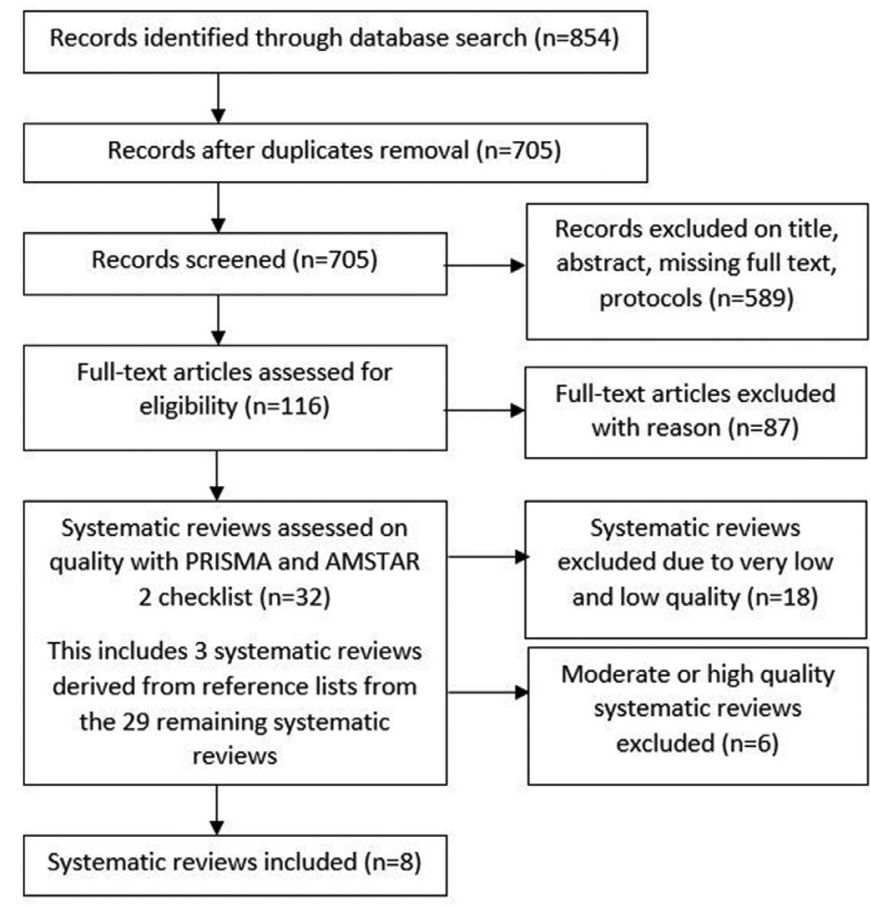

Figure 1 Flow diagram of studies found, assessed, excluded and included. AMSTAR, A Measurement Tool to Assess Systematic Reviews; PRISMA, Preferred Reporting Items for Systematic Reviews and MetaAnalyses.

that good outcome is a result of a structure that promotes a good process, ${ }^{19}$ and that any efforts to improve the approaches in the triad must be seen in the light of care and needs of the patients. ${ }^{20}$ In the ECHO model, the outcomes of medical care are classified along three general dimensions: clinical, economic and humanistic. ${ }^{21}$ The assertion of this study is that: healthcare professionals with their education and skills represent the structure. The process represents what is done in the patient-healthcare professional interaction (eg, interventions to improve medication adherence), and the outcome is the clinical, humanistic and economic outcomes measured because of the adherence intervention.

With this background, the aim of the study was to present evidence for healthcare-provided medication adherence interventions on clinical, economic and humanistic outcomes among patients.

\section{METHODS}

\section{Literature search}

After initial search, a search strategy was built using relevant text words and their synonyms, and opposites, in addition to terms found going through the controlled vocabulary in the databases: MeSH (in Medline), Emtree (in Embase) and

\begin{tabular}{lll}
\hline Table 1 & Results from AMSTAR 2 & checklist evaluation \\
\hline AMSTAR 2 score & Interpretation & $\begin{array}{l}\text { Number of systematic reviews } \\
\text { excluded or included }\end{array}$ \\
\hline $0-4$ & Critically low & Excluded $(\mathrm{n}=3)$ \\
$5-8$ & Low & Excluded $(\mathrm{n}=15)$ \\
$9-11$ & Moderate & Excluded $(\mathrm{n}=4) ;$ included $(\mathrm{n}=4)$ \\
$12-16$ & High & Excluded $(\mathrm{n}=2) ;$ included $(\mathrm{n}=4)$ \\
\hline
\end{tabular}

AMSTARM, A Measurement Tool to Assess Systematic Reviews.
CINAHL Subject Headings (in CINAHL). The final searches (performed 22 December 2017) were restricted to year published (2007-2017), English language and systematic reviews. A detailed description of the search is given in online supplementary appendix 1 . The Cochrane Library itself was not searched. Relevant articles found in the initial search were crosschecked for ensuring that they also were included in the final search strategy. Articles were screened for inclusion and exclusion criteria on title and abstract. Full text was assessed on articles not excluded by title and abstract. The purpose of the literature search was to find systematic reviews that matched the inclusion criteria. If all the studies in a systematic review did not match, individual studies in the systematic review were extracted. Also references from the systematic reviews selected for quality assessment were screened.

\section{Inclusion criteria}

Included studies were systematic reviews that contained medication adherence interventions, and one or more of the predefined outcomes (mortality, morbidity, healthcare utilisation, healthcare costs and patient satisfaction/quality of life). The systematic review had to include at least two randomised controlled trials (RCT) that matched the inclusion criteria. The RCTs extracted from systematic reviews had to have a sample size of at least 60 participants in each group to have enough power to detect an absolute difference. ${ }^{10}$ Finally, the studies must be evaluated as of moderate or high in methodological quality.

\section{Exclusion criteria}

Systematic reviews only reporting medication adherence, surrogate endpoints and not the predefined hard endpoints were excluded. Studies had to have a description of medication adherence interventions, not only results of interventions. Studies where the interventions were very complex and had multiple components not only regarding medication adherence (eg, diet, exercise, and so on) were excluded. Studies were also excluded if not all participants used medication. Cochrane reviews targeting several mixed components, and not only medication adherence, were excluded if they reported low or very low evidence (Grading of Recommendations Assessment, Development and Evaluation) by the authors, even if they scored high on A Measurement Tool to Assess Systematic Reviews (AMSTAR) 2 and Preferred Reporting Items for Systematic Reviews and Meta-Analyses (PRISMA).

\section{Assessment of study quality}

PRISMA and AMSTAR 2 are validated tools for assessing quality of systematic reviews. They were used according to instructions $^{22} 23$ on 32 systematic reviews. Ten of them were also evaluated by a coresearcher for validation. The PRISMA checklist was used to assess the reporting characteristics, meaning the quality of reporting, and the AMSTAR 2 checklist was used to assess the methodological quality of the systematic reviews. Methodological quality was given highest weight for studies to be included, only studies with moderate to high quality were included.

PRISMA is a reporting guidance with a 27 -item checklist. The PRISMA statement is used by authors to ensure a clear, complete and transparent systematic review. ${ }^{22}$ The checklist contains seven sections/topics with the associated questions. The answers were given very much rigour as to where in the systematic reviews they were found, meaning that they had to 
Table 2 Included systematic review with PRISMA and AMSTAR 2 score

\begin{tabular}{|c|c|c|c|c|}
\hline Systematic review & $\begin{array}{l}\text { PRISMA } \\
\text { score } \\
2 \text { evaluators }\end{array}$ & $\begin{array}{l}\text { Consensus } \\
\text { PRISMA } \\
\text { score }\end{array}$ & $\begin{array}{l}\text { AMSTAR } 2 \\
\text { score } \\
2 \text { evaluators }\end{array}$ & $\begin{array}{l}\text { Consensus } \\
\text { AMSTAR } 2 \\
\text { score }\end{array}$ \\
\hline Al-Aqeel et $a^{30 *}$ & $23 / 20$ & 24 & $12 / 10$ & 12 \\
\hline Mathes et $a l^{31}$ & $20 / 20$ & 21 & $10.5 / 9.5$ & 10.5 \\
\hline Ngwatu et $a^{32}$ & $19 / 16$ & 21 & $9 / 7$ & 9 \\
\hline Nieuwlaat et $a /^{10 *}$ & $22 / 17$ & 21 & $11 / 9.5$ & 10 \\
\hline Normansell et $a^{33 *}$ & $26 / 24$ & 26 & $15 / 13.5$ & 15 \\
\hline Weeks et $a l^{34 *}$ & $25 / 24$ & 25 & $14.5 / 13.5$ & 15 \\
\hline Zaugg et $a^{35 *}$ & $23 / 17$ & 22 & $13 / 9.5$ & 13 \\
\hline Zhong et $a^{36}$ & $23 / 20$ & 23 & $9.5 / 11$ & 9.5 \\
\hline
\end{tabular}

${ }^{*}$ Cochrane reviews.

AMSTAR, A Measurement Tool to Assess Systematic Reviews; PRISMA, Preferred

Reporting Items for Systematic Reviews and Meta-Analyses.

be found where they were supposed to be due to the checklist. The answer 'yes' was denoted as page number, figure or table where the item was found. A 'no' or 'not applicable' was denoted as $\div$. When summarising, all the 'yes' was given 1 point, making the potential total score 27 .

AMSTAR was published in $2007,{ }^{24}$ and has been widely used for assessing methodological quality of systematic reviews ${ }^{25-27}$ of both Cochrane and non-Cochrane overviews of healthcare interventions. ${ }^{28}$ AMSTAR was recently updated to AMSTAR 2, and now consists of a 16-item online tool for evaluating systematic reviews as high, moderate, low or critically low of methodological quality. ${ }^{24}$ Although AMSTAR 2 was not intended to generate an overall score, it was used with that purpose for this report, to find the systematic reviews with the highest quality. Based on the original AMSTAR tool, a score range was made for ranking the systematic reviews into critical low (0-4 p), low (5-8), moderate (9-11) and high (12-16). Two researchers evaluated the studies separately. Any disagreement was resolved through discussion to reach consensus about the final score.

\section{Summarising evidence}

The evidence is based on the data that were presented in the systematic reviews for each of the included RCTs. Individual RCTs were not assessed and evaluated through the primary sources, only from the systematic reviews they were included in.

The evidence of the interventions was interpreted on the reported CIs and the p values of the RCTs in the systematic reviews. Studies were evaluated based on statically significant results on the predefined outcomes. For risk ratio, relative risk,
OR, risk difference and relative rate the 'no effect' cut-off was set at 1 , meaning that if the CIs crossed 1 , the interpretation was that the results were not statistically significant. For absolute risks and mean differences, the 'no effect' cut-off was set on 0 , meaning the interpretation was not statistically significant if 0 was within the CI of the results. ${ }^{29}$

\section{RESULTS}

\section{Literature search and inclusion}

The database search identified a total of 854 studies (figure 1). After initial screening and full-text reading 32 studies remained for inclusion before quality assessment. A detailed description of studies excluded before quality assessment is provided in online supplementary appendix 2 .

\section{Quality assessments}

Many of the systematic reviews did not have a clear PICO (population, intervention, comparator group, outcome) in their research questions or inclusion criteria. This could be because the studies included in the systematic reviews already had elements of it, for example, RCTs having control groups. An accessible protocol was missing in many systematic reviews. In the PRISMA checklist, studies received a positive score when information was provided about the existence of a protocol, but if the protocol was not found (searching PROSPERO database), there was consequently lack of score in the AMSTAR 2 checklist. There was also a lack of a clear explanation of which study designs that were selected in most of the systematic reviews. Many of the systematic reviews did not provide a full search strategy and did not search for grey literature. Almost all systematic reviews reported that study selection and extraction were done in duplicate. Most of the systematic reviews not being Cochrane reviews did not provide a list of excluded studies and the reason for exclusion. The Cochrane reviews had the most detailed description of the studies included. Almost every systematic review described a satisfactory technique for assessing risk of bias in individual studies but there was often a lack of reporting funding of individual studies. Consequently, systematic reviews including meta-analysis were given a higher score due to the amount of questions addressing meta-analysis. Heterogeneity was reported in most of the SR because of its nature in the intervention and measurements of outcome. Overall there was reported funding of systematic reviews and conflict of interests. As presented in table 1, more than half of the systematic reviews assessed had a low or critically low quality. Fourteen had moderate to high quality, and eight of those were selected for inclusion and consensus assessment. A detailed description

Table 3 Overview of outcomes extracted from the RCTs in the included systematic reviews

\begin{tabular}{|c|c|c|c|c|c|}
\hline Systematic review & Mortality & Morbidity & Healthcare utilisation & Healthcare costs & QoL/patient satisfaction \\
\hline Al-Aqeel et $a l^{30 *}$ & & $\checkmark$ & & & $\checkmark$ \\
\hline Mathes et $a l^{31}$ & $\checkmark$ & $\checkmark$ & & & $\checkmark$ \\
\hline Ngwatu et al ${ }^{32}$ & $\checkmark$ & $\checkmark$ & & & \\
\hline Nieuwlaat et $a l^{10 *}$ & $\checkmark$ & $\checkmark$ & $\checkmark$ & & $\checkmark$ \\
\hline Normansell et $a^{33 *}$ & & $\checkmark$ & $\checkmark$ & $\checkmark$ & $\checkmark$ \\
\hline Weeks et $\left.a\right|^{34 *}$ & & $\checkmark$ & $\checkmark$ & $\checkmark$ & $\checkmark$ \\
\hline
\end{tabular}

QoL, quality of life; $\mathrm{RCT}$, randomised controlled trial. 
Table 4 Summary of adherence intervention type and effects on outcomes. Based on detailed description of each study in online supplementary appendix 5

\begin{tabular}{|c|c|c|c|c|c|c|}
\hline Intervention type & Mortality & Morbidity & Healthcare utilisation & Healthcare costs & Patient satisfaction & QoL \\
\hline Reminders & 4 RCTs & 5 RCTs & 2 RCTs & & 2 RCTs & 2 RCTs \\
\hline Simplified dosing & & 2 RCTs & 2 RCTs & & 2 RCTs & $3 \mathrm{RCTs}$ \\
\hline $\begin{array}{l}\text { Direct observed treatment } \\
\text { (DOT) }\end{array}$ & $1 \mathrm{RCT}$ & $1 \mathrm{RCT}$ & & & & \\
\hline Patient education & 2 RCTs & 3 RCTs & 7 RCTs & $1 \mathrm{RCT}$ & $1 \mathrm{RCT}$ & $8 \mathrm{RCTs}$ \\
\hline Counselling & $1 \mathrm{RCT}$ & 2 RCTs & $1 \mathrm{RCT}$ & & $1 \mathrm{RCT}$ & 3 RCTs \\
\hline Web-based programmes & & & $1 \mathrm{RCT}$ & & & \\
\hline Prescribing pharmacists & & $1 \mathrm{RCT}$ & $1 \mathrm{RCT}$ & & & $3 \mathrm{RCTs}$ \\
\hline $\begin{array}{l}\text { Physicians assess to } \\
\text { adherence information }\end{array}$ & & 2 RCTs & 2 RCTs & & & \\
\hline All RCTs positive result & $\begin{array}{l}2 \text { RCTs positive and } \\
1 \mathrm{RCT} \text { negative result }\end{array}$ & $\begin{array}{c}\text { RCTs } 50 / 50 \text { positive/ } \\
\text { negative result within or } \\
\text { between }\end{array}$ & $\begin{array}{l}3 \text { RCTs positive and } \\
5 \text { RCTs negative result }\end{array}$ & $\begin{array}{c}2 \text { RCTs negative, } \\
1 \mathrm{RCT} \text { positive, or } \\
1 \mathrm{RCT} \text { negative } \\
\text { and } 1 \mathrm{RCT} \text { positive/ } \\
\text { negative result } \\
\text { within }\end{array}$ & \multicolumn{2}{|c|}{ All RCTs negative result } \\
\hline
\end{tabular}

QoL, quality of life; RCT, randomised controlled trial.

of the studies excluded after quality assessment is provided in online supplementary appendix 3.

Table 2 gives the PRISMA and AMSTAR 2 scores from two evaluators and the consensus scores. A detailed description of the result of the assessment is given in online supplementary appendix 4.

\section{Summary of evidence}

The eight systematic reviews included in this report were published between 2014 and 2018. Five of them were Cochrane reviews. Thirty-seven RCTs published between 2000 and 2016 involving 28600 patients were extracted from the systematic reviews. The studies included mostly adult patients of various ages. The group sample size ranged from $\mathrm{n}=63$ to $\mathrm{n}=3260$. Follow-up time ranged from 12 weeks to 2 years. Table 3 shows an overview of the outcomes reported in the RCTs extracted from the systematic reviews. Characteristics of each systematic review and the results of the RCTs extracted from them are presented and tabulated in online supplementary appendix 5 .

The results from the tables representing the RCTs from each systematic review, presented in detail in online supplementary appendix 5 , are summarised across the systematic reviews, representing intervention type and outcome type. Table 4 shows the studies where a positive, negative or mixed effect on outcomes was reported. This table does not include the intervention effect on adherence. These are presented in online supplementary appendix 5. A detailed description of the results based on intervention type is given in online supplementary appendix 6 and based on effects on outcomes in online supplementary appendix 7. A detailed reference list is given in online supplementary appendix 8 .

The most frequent intervention type reported was patient education, delivered by pharmacists or nurses. Improvement in adherence was reported in most of the studies where patient education was provided. For counselling there was improvement 
in half of the studies. In studies where the intervention was delivered by pharmacists who prescribed medication, it was uncertain whether the intervention had any effect on adherence. In all the studies where physicians were the interventionists, the intervention failed to show improvement in adherence.

Reminder interventions, direct observed treatment, web-based programmes and interventions performed by physicians failed to show statistically improvements in outcomes. Simplified dosing, patient education and counselling with and without pharmacists prescribing showed mixed effects among the outcomes. There was evidence that patient education had a positive effect on morbidity and patient satisfaction. Counselling had a positive effect on mortality and healthcare utilisations. Morbidity was also reduced when pharmacists prescribed medication. Since most of these improvements were based on the results of only one RCT, the evidence is weak.

\section{DISCUSSION}

The results of the studies with counselling intervention showed a reduction in mortality and healthcare utilisation, and improvements in adherence were mixed. Patient education showed an increased patient satisfaction and some benefits on mortality and healthcare utilisation, while patient education in addition to pharmacist prescribing medication showed decreased morbidity. Educational intervention had mostly a positive effect on adherence. Simplification of dose showed some improvement in morbidity and patient satisfaction, and a mixed effect on adherence. However, no intervention was shown effective across all the outcomes of interest in this study, indication that choice of intervention should be in concordance with the type of challenges the patient has with adherence.

How the adherence was measured is not without importance, as the methods can have unintended impact on the results, for example, under-reporting or over-reporting in patient self-reports, electronic medication monitors that record opening of the medication container but do not in fact record if the medication is being taken by the patient, and so on. However, this was not the focus in this report and is not reported.

There are several limitations in this study. Only systematic reviews that achieved a moderate to high AMSTAR 2 score were included in this report. There could have been individual studies of high quality within the lower quality systematic reviews that were missed because of the quality assessment. A major limitation is that bias in the individual RCTs was not assessed. However, bias has been assessed by the authors of the systematic reviews. There was some degree of incomplete reporting of data. This could be caused by selective reporting, but it was not examined.

The literature search for this report was restricted to systematic reviews published the last 10 years (2007-2017), although two systematic reviews from 2018 were included that were found by a non-comprehensive Google search of systematic reviews published in 2018. Language was restricted to English, so this report is prone to language bias, with a chance of missing potential studies published in non-English language journals. This search did not aim for unpublished studies ('grey literature'), but some of the systematic reviews had included unpublished studies in them.

Some studies may have been excluded because of indistinct reporting of the intervention. Studies reporting only 'health care professionals' as interventionists were excluded. Because this report was restricted to interventionists being pharmacists, nurses or clinicians, the results of this report may be skewed against middle or high-income countries, since low-income countries were more likely to use non-healthcare professionals as lay or community workers as interventionists.

There was no systematic review that gave a full match with the aim and inclusion criteria for this report, instead individual RCTs that matched the inclusion criteria were extracted from the systematic reviews. Likewise, meta-analysis in the systematic reviews could not be used, making the power in clinically and statistically significance weaker as single RCTs were extracted. Overall the studies were heterogeneous in population type, intervention type and length, and which outcomes that were measured. There was a considerable difference in how the adherence was measured, and what was considered 'good adherence'. Some of the studies were complex and had several components in their intervention, because of this there was a degree of overlap between the studies, so it was difficult to divide them in a rigid manner.

Another limitation by using individual studies from systematic reviews, and not the primary source itself, is the possibility of misreading by the authors of the systematic reviews. Any interpretation, correct or incorrect, will follow further if the author is not interpreting the result from the individual studies by him or herself. The primary studies were added so they can be found, they were not assessed by the author of this report.

This study was planned as an MSc project in Pharmacy. Three students performed similar studies on different interventions aimed at improving important outcomes. In this study, the student (NCW) performed all activities supervised by TE. The quality assessment was also performed by one costudent researcher. As this report was mainly conducted by a student, not having the same amount of expertise and knowledge in the field as professional researches have, it is more exposed to flaws. The researcher did not have any training in quality assessment of systematic reviews before the work with this report was performed but gained experience through the process. This could have caused some variation in how the first quality assessments were considered compared with the last quality assessment. However, consistency was strived for, and it involved changes in some of the previous quality assessments.

This report can be interesting for several readers. For healthcare decision makers, this report gives an insight into which interventions and interventionists have shown to improve adherence and/or clinical, economic and humanistic outcomes or not. In other words, what type of intervention resources are reasonable to invest in. For researchers who are planning to do similar studies, new studies can be based on the studies in this report. For healthcare professionals who want to enhance their awareness and knowledge about adherence interventions, and want to use them in their practices. Finally, this report is also interesting for patients and consumers of medications by gaining awareness of the challenges insufficient adherence can lead to.

\section{CONCLUSION}

Some interventions were found to have positive effect on adherence and outcomes, but no single strategy showed improvement in all settings. For future research, patients should be screened for non-adherence to reveal both if they are non-adherent and type of non-adherence, as well as bigger sample sizes and longer duration of follow-up.

Contributors This study was planned as an MSc project in Pharmacy. The student (NCW) performed all activities supervised by TE in MSc report. Based on the MSC report we have written this manuscript together.

Funding The authors have not declared a specific grant for this research from any funding agency in the public, commercial or not-for-profit sectors. 
Competing interests None declared.

Patient consent Not required.

Provenance and peer review Not commissioned; externally peer reviewed.

\section{REFERENCES}

1 Mulrow CD. Systematic Reviews: Rationale for systematic reviews. BMJ 1994;309:597-9.

2 Sackett DL, Rosenberg WM, Gray JA, et al. Evidence based medicine: what it is and what it isn't. BMJ 1996;312:71-2.

3 Wiffen P, Eriksson T, Lu H. CHAPTER 2 introduction to evidence-based practice in evidence-based pharmacy 2nd edition. Eur J Hosp Pharm 2013;20:324-7.

4 Eriksson T, Lu H, Wiffen P. Chapter 6: How to best practice evidence-based pharmacy with your available resources? Eur J Hosp Pharm 2014;21:194-201.

5 Wiffen P, Eriksson T, Lu H. CHAPTER 3: Asking and formulating the right questions and finding useful resources in evidence-based pharmacy_2nd edition. Eur J Hosp Pharm Sci 2014;21:2-6.

6 WHO. The World Medicines Situation 2011. Geneva: World Health Organization, 2011.

7 Tarn DM, Heritage J, Paterniti DA, et al. Physician communication when prescribing new medications. Arch Intern Med 2006;166:1855-62.

8 Horne R, Weinman J, Barber N, et al, 2005. Concordance, Adherence and Compliance in Medicine Taking. Report for the National Co-ordinating Centre for NHS Service Delivery and Organisation R \& D (NCCSDO). http://www.netscc.ac.uk/hsdr/files/ project/SDO_FR_08-1412-076_V01.pdf (verified 18 Jun 2018).

9 WHO. Adherence to Long-term Therapies: Evidence for Action: World Health Organization, 2003.

10 Nieuwlaat R, Wilczynski N, Navarro T, et al. Interventions for enhancing medication adherence. Cochrane Database Syst Rev 2014;11:Cd000011.

11 Claxton AJ, Cramer J, Pierce C. A systematic review of the associations between dose regimens and medication compliance. Clin Ther 2001;23:1296-310.

12 Jimmy B, Jose J. Patient medication adherence: measures in daily practice. Oman Med J 2011;26:155-9.

13 Granger BB, Swedberg K, Ekman I, et al. Adherence to candesartan and placebo and outcomes in chronic heart failure in the CHARM programme: double-blind, randomised, controlled clinical trial. Lancet 2005;366:2005-11.

14 Rasmussen JN, Chong A, Alter DA. Relationship between adherence to evidencebased pharmacotherapy and long-term mortality after acute myocardial infarction. JAMA 2007;297:177-86.

15 Simpson SH, Eurich DT, Majumdar SR, et al. A meta-analysis of the association between adherence to drug therapy and mortality. BMJ 2006;333:15.

16 Roebuck MC, Liberman JN, Gemmill-Toyama M, et al. Medication adherence leads to lower health care use and costs despite increased drug spending. Health Aff 2011:30:91-9.

17 Dragomir A, Côté R, Roy L, et al. Impact of adherence to antihypertensive agents on clinical outcomes and hospitalization costs. Med Care 2010;48:418-25.

18 Donabedian A, Rashid B. An introduction to quality assurance in health care. United States: Oxford University Press, 2002.

19 Ameh S, Gómez-Olivé FX, Kahn K, et al. Relationships between structure, process and outcome to assess quality of integrated chronic disease management in a rural
South African setting: applying a structural equation model. BMC Health Serv Res 2017;17:229.

20 Ayanian JZ, Markel H. Donabedian's Lasting Framework for Health Care Quality. N Engl J Med 2016;375:205-7.

21 Kozma CM, Reeder CE, Schulz RM. Economic, clinical, and humanistic outcomes: a planning model for pharmacoeconomic research. Clin Ther 1993; 15:1121-32.

22 Liberati A, Altman DG, Tetzlaff J, et al. The PRISMA statement for reporting systematic reviews and meta-analyses of studies that evaluate healthcare interventions: explanation and elaboration. BMJ 2009;339:b2700.

23 Shea BJR, Wells BC, Thuku G, et al. AMSTAR 2: a critical appraisal tool for systematic reviews that include randomised or non-randomised studies of healthcare interventions, or both. Ottawa: AMSTAR team, 2017.

24 Shea BJ, Reeves BC, Wells G, et al. AMSTAR 2: a critical appraisal tool for systematic reviews that include randomised or non-randomised studies of healthcare interventions, or both. BMJ 2017;358:j4008.

25 Shea BJ, Grimshaw JM, Wells GA, et al. Development of AMSTAR: a measurement too to assess the methodological quality of systematic reviews. BMC Med Res Methodol 2007:7:10.

26 Shea BJ, Hamel C, Wells GA, et al. AMSTAR is a reliable and valid measurement tool to assess the methodological quality of systematic reviews. J Clin Epidemiol 2009;62:1013-20.

27 Pollock M, Fernandes RM, Becker LA, et al. What guidance is available for researchers conducting overviews of reviews of healthcare interventions? A scoping review and qualitative metasummary. Syst Rev 2016;5:190.

28 Pollock M, Fernandes RM, Hartling L. Evaluation of AMSTAR to assess the methodological quality of systematic reviews in overviews of reviews of healthcare interventions. BMC Med Res Methodol 2017;17:48.

29 McCormack J, Vandermeer B, Allan GM. How confidence intervals become confusion intervals. BMC Med Res Methodol 2013;13:134

30 Al-Aqeel S, Gershuni O, Al-Sabhan J, et al. Strategies for improving adherence to antiepileptic drug treatment in people with epilepsy. Cochrane Database Syst Rev 2017;2:CD008312.

31 Mathes T, Antoine SL, Pieper D. Adherence-enhancing interventions for active antiretroviral therapy in sub-Saharan Africa: a systematic review and meta-analysis. Sex Health 2014;11:230-9.

32 Ngwatu BK, Nsengiyumva NP, Oxlade 0, et al. The impact of digital health technologies on tuberculosis treatment: a systematic review. Eur Respir J 2018;51:1701596.

33 Normansell R, Kew KM, Stovold E. Interventions to improve adherence to inhaled steroids for asthma. Cochrane Database Syst Rev 2017;4:Cd012226.

34 Weeks G, George J, Maclure K, et al. Non-medical prescribing versus medical prescribing for acute and chronic disease management in primary and secondary care. Cochrane Database Syst Rev 2016;11:Cd011227.

35 Zaugg V, Korb-Savoldelli V, Durieux P, et al. Providing physicians with feedback on medication adherence for people with chronic diseases taking long-term medication. Cochrane Database Syst Rev 2018;1:CD012042.

36 Zhong $\mathrm{H}, \mathrm{Ni}$ XJ, Cui M, et al. Evaluation of pharmacist care for patients with chronic obstructive pulmonary disease: a systematic review and meta-analysis. Int J Clin Pharm 2014;36:1230-40. 